\title{
ESI-IMS-QTOF: a regioselective Michael addition study
}

\author{
Pedro H. P. R. Carvalho, ${ }^{a}$ Alexandre F. Gomes, ${ }^{b}$ Luciana M. Ramos, ${ }^{a}$ Fábio C. \\ Gozzo*b $^{\star b}$ Eufrânio N. da Silva Júnior*c and Brenno A. D. Neto*a \\ ${ }^{a}$ Institute of Chemistry, University of Brasília, Brazil. ${ }^{b}$ Institute of Chemistry, State University of Campinas \\ Brazil. 'Institute of Exact Sciences, Department of Chemistry, Federal University of Minas Gerais, Brazil.
}

*e-mail: eufranio.junior@yahoo.com.br, fabio@iqm.unicamp.br, brenno.ipi@gmail.com

Keywords: Michael addition, ion mobility, mass spectrometry

\section{INTRODUCTION}

1,2- and 1,4- additions of nucleophiles to $\alpha, \beta-$ unsaturated carbonyl compounds are among the most useful transformations in organic synthesis. 1,4-Naphthoquinones have been reported as important active compounds and are interesting substrates for studying the regioselectivity of a nucleophilic addition reaction. ${ }^{1}$ Lately, the ion mobility spectrometry (IMS) may become an important approach to study reaction mechanisms. ${ }^{2}$

Herein, we describe ESI and IMS as tools in the study of the 1,2 vs. 1,4-regioselective addition of amines to 2-bromo-1,4-naphthoquinone.

\section{RESULTS AND DISCUSSION}

Upon mixing the reagents, the reaction mixture was analyzed by ESI-IMS-QTOF after $15 \mathrm{~min}$. Two more intense signals of $m / z 306$ (and 308) and $m / z 228$ were noted. It is interesting that no signal of $m / z 308$ (and 310) of the direct addition of pyrrolidine (2) to the bromoquinone $\mathbf{1}$ was detected. The other important signal observed is that of $m / z 228$, attributed to the direct loss of $\mathrm{HBr}$ from the intermediates $\mathbf{3}$ and/or $\mathbf{4}$, as seen in Scheme 1.

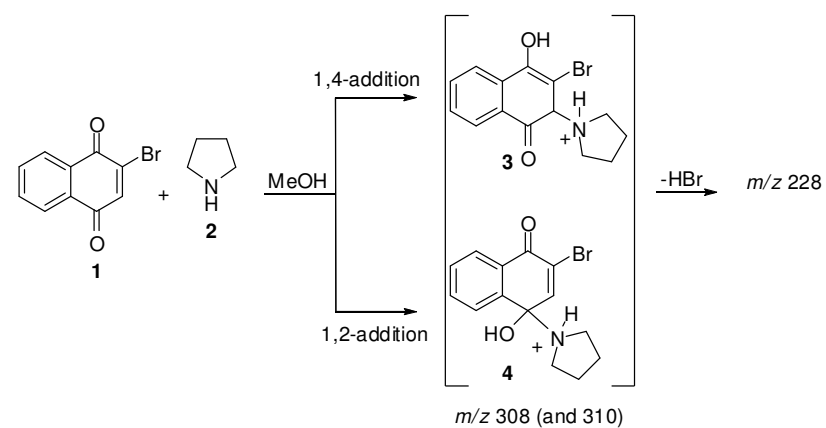

Scheme 1. 1,2- and 1,4- additions of pyrrolidine to 2bromo-1,4-naphthoquinone.

The suggested formation of the ion of $\mathrm{m} / \mathrm{z} 228$ derived from the 1,4-addition occurs from two different pathways that lead to the same ion, independently if the addition takes place at the bromine-containing carbon or at the adjacent carbon.
The proposed formation of the ion of $m / z 228$ derived from 1,2-addition can be occurs by the direct attack of amine at the carbonyl group provides protonated specie that can produce a fivemembered ring cyclic and after $\mathrm{HBr}$ eliminates the fragment ion $\mathrm{m} / \mathrm{z} 228$ can be observed.

Envisaging the separation of the structural isomers 3 and $\mathbf{4}$, the signal of $\mathrm{m} / \mathrm{z} 228$ was submitted to IMS. The chromatogram is showed in Figure 2.

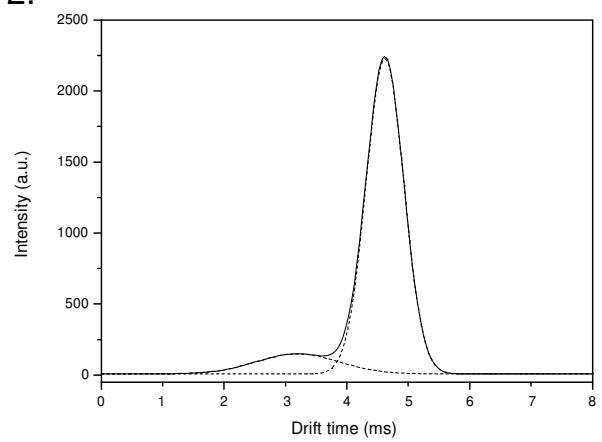

Figure 2. The signal of $m / z 228$ separated by IMS analysis.

Considering the areas of the graphics it is possible to quantify the relation among both isomers. The ratio of Area $A$ (right, 1704.81 a.u.) /Area B (left, 238.37 a.u.) was 7.15 indicating the proportion of $7: 1$ favoring the regioisomer from the 1,4-addition.

\section{CONCLUSION}

It has been demonstrated that IMS and ESI-QTOF analyses are a powerful combination to study the regioselectivity addition in systems such as quinone derivatives. The 1,4-addition of pyrrolidine is preferred to 2-bromo-1,4-naphthoquinone system.

\section{ACKNOWLEDGEMENTS}

This research was supported by grants from the CNPq, CAPES, FAPDF, FAPESP, UFMG and DPP.

\section{REFERENCES}

${ }^{1}$ Zhou, W. M.; Liu, H.; Du, D. M. Org. Lett. 2008, 10, 13.

${ }^{2}$ Kanu, A. B.; Dwivedi, P.; Tam, M.; Matz, L.; Hill, H. H. J. Mass Spectrom. 2008, 43, 1. 